\title{
Multimodal sequential treatment for occluded TIPS: Case report and review of literature
}

\author{
Nicoletta De Matthaeis ${ }^{1, *}$, Carmine Di Stasi ${ }^{2}$,* Fabrizio Pizzolante', Riccardo Manfredi ${ }^{2}$, Gian Ludovico Rapaccini', \\ and Luca Miele ${ }^{1}$
}

'Area Medicina Interna, Gastroenterologia ed Oncologia Medica. Policlinico A. Gemelli IRCCS, Università Cattolica del S. Cuore, Roma; ${ }^{2}$ Area Diagnostica per Immagini. Policlinico A. Gemelli IRCCS, Università Cattolica del S. Cuore, Roma, Italy

Dear Editor,

Transjugular intrahepatic portosystemic shunt (TIPS) is an established treatment for refractory ascites as well as variceal bleeding. ${ }^{1,2}$ Unfortunately, shunt dysfunction (stenosis/occlusion) commonly occurs in $13 \%$ to $50 \%$ of TIPS patients, depending on the stent type (covered or bare metal) placed. We report a case of occluded TIPS with splenic and superior mesenteric veins (SMV) thrombosis, which was managed with mechanical and suction thrombectomy, following angioplasty and stenting.

A 64-year-old hepatic cryptogenic cirrhosis male patient, complicated by portal hypertension and refractory ascites was treated in 2011 by placement of Viatorr TIPS Endoprosthesis (W.L. Gore \& Associates, Newark, DE, USA; graft length lined $7 \mathrm{~cm}$ and unlined $2 \mathrm{~cm}$, internal diameter $10 \mathrm{~mm}$ ). On March 2018 he was referred to the authors' hospital because of progressive increase of weight and abdominal circumference associated to dyspnea and lower limbs edema. The patient was in Child-Pugh B8 class, with normal white blood cell $\left(5.23 \times 10^{9} / \mathrm{L}\right)$ and platelet count $\left(155 \times 10^{9} / \mathrm{L}\right)$.

The annual routine Doppler ultrasound (US) performed three months before showed an evidently expanded stent between the right portal and right hepatic vein, with regular peak shunt velocity. The main portal vein flow velocity and directionality in the in- trahepatic portal veins were normal. Abdominal US at the authors' hospital, revealed an absent flow within the shunt, more precisely at the mid-shunt, the portal and hepatic venous end. Additionally, large ascites with marked abdominal distension was evident. Abdominal computed tomography angiography confirmed a subacute thrombosis of the main portal trunk, which extended to the right portal vein, and spleno-mesenteric confluence (Fig. 1).

The occluded TIPS was navigated using $5 \mathrm{Fr}$ catheter and guidewire via transjugular approach, and venography confirmed complete occlusion of TIPS and thrombosis in main portal vein (Fig. 2). Initially, mechanical thrombectomy was performed using $10 \mathrm{Fr}$ Aspirex S system (Straub Medical AG, Wangs, Switzerland) (Fig. 3A). A partial outflow within the shunt with residual thrombosis persistence in spleno-mesenteric confluence and decreased caliber was demonstrated with portal venogram images after the thrombectomy and aspiration procedure. Therefore an $8 \mathrm{~mm}$ and $10 \mathrm{~mm}$ balloon angioplasty was performed in the shunt and spleno-mesenteric confluence up to the main portal vein. A post balloon angioplasty venography still revealed an unsatisfying flow. Therefore four E-Luminexx 12×40 mm stents (Bard GmbH/Angiomed, Karlsruhe, Germany) were placed: two from the spleno-

\begin{abstract}
Abbreviations:
CTA, computed tomography angiography; MT, mechanical thrombectomy; PT, pharmacologic thrombolysis; SMV, superior mesenteric veins; TIPS, tansjugula intrahepatic portosystemic shunt; US, ultrasound

*These authors contributed equally to this work.
\end{abstract}

\section{Corresponding author : Luca Miele}

Area Medicina Interna, Gastroenterologia ed Oncologia Medica, Fondazione Policlinico Universitario A. Gemelli IRCCS, Università Cattolica del Sacro Cuore, 8, Largo Gemelli, Roma 00168, Italy

Tel: +39-6-30155451, Fax: +39-6-35502775

E-mail: luca.miele@policlinicogemelli.it or luca.miele@unicatt.it https://orcid.org/0000-0003-3464-0068 


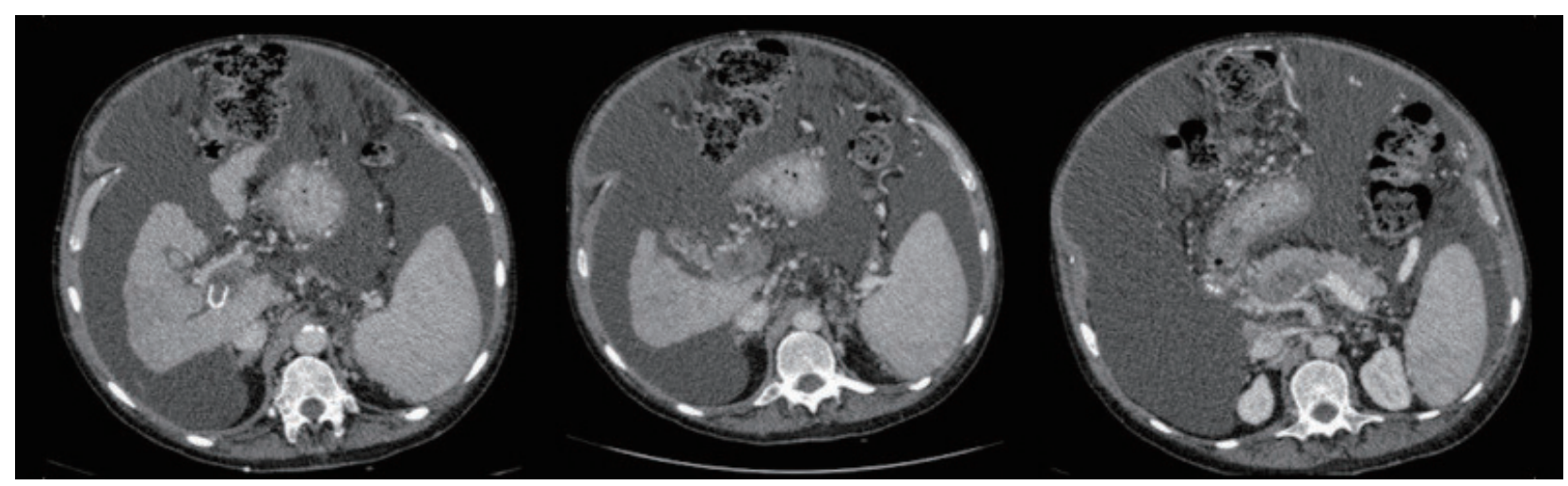

Figure 1. Subacute thrombosis of the main portal trunk extended to the bifurcation and the right branch and to the spleno-mesenteric confluence.

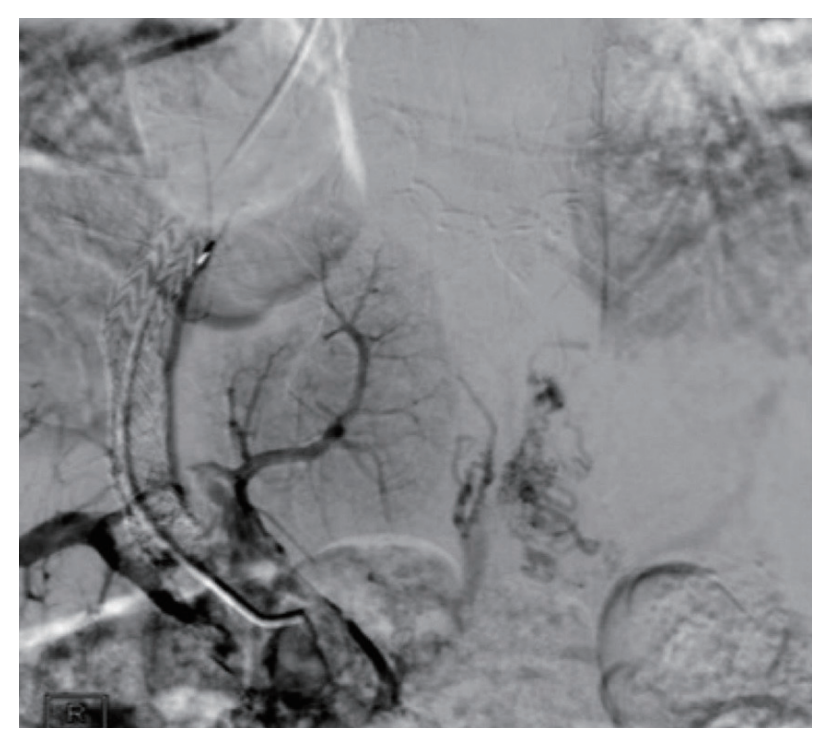

Figure 2. Venography from a transjugular trans TIPS approach showing occlusion of TIPS extending into the portal vein, splenic vein and the superior mesenteric vein. TIPS, tansjugular intrahepatic portosystemic shunt.

mesenteric confluence up to the main portal vein and additional two within the shunt. The portal venogram images demonstrated a rapid contrast medium outflow toward the shunt and the hepatic veins (Fig. 3B). Anticoagulant treatment with fondaparinux sodium, $5 \mathrm{mg}$ subcutaneously once daily was administered for 30 days.

An US evaluation, performed 1 month after TIPS revision, showed stent patency between the right portal and hepatic vein, in the distal tract of the splenic vein, which extended to the spleno-mesenteric confluence with a regular peak shunt velocity. A minimal thrombosis with a regular blood flow direction and velocity of the spleno-mesenteric confluence, the distal tract of the main portal vein extended to the bifurcation and to the right branch of the portal trunk was revealed. Seven months after the intervention, US and CT scan demonstrated a complete thrombosis resolution of the shunt, intrahepatic portal vein branches and the main portal trunk up to the spleno-mesenteric confluence (Fig. 4).

Technical variables during TIPS creation, thrombophilic risk factors, hypercoagulable state and/or large competitive spontaneous splenorenal shunts, determining flow reduction in TIPS, can possibly cause stent occlusion. Furthermore, Yue-Meng et al. ${ }^{3}$ demonstrated that low white blood cell count, high Child Pugh class and severe ascites are independent predictors of portal vein thrombosis in TIPS-treated patients with cirrhosis. An early detection and correction of TIPS failure is crucial. The most commonly applied technique for TIPS occlusion is recanalization performed through the jugular vein with a hydrophilic guide wire or a Cook needle (Colapinto or Rosch-Uchida), ${ }^{4}$ although the access to the shunt is sometimes difficult. ${ }^{4}$ Therefore, alternative therapeutic strategies ${ }^{5}$ could be: 1) fluoroscopically guided transhepatic stent puncture, 2) creation of a new shunt via direct cavoportal puncture or with the so-called 'gun-sight approach' ${ }^{\text {'6 }}$ or 3) creation of a new parallel shunt?

Mechanical thrombectomy (MT) or pharmacologic thrombolysis, directly through the shunt, can manage thrombotic occlusion and stenosis of TIPS and of the portal venous system. Balloon and suction embolectomy, basket extraction of clots and other mechanical thrombectomy with special devices are reported MT techniques ${ }^{8}$ which may be used to reopen the shunt and the thrombosed venous system. These MT techniques can be performed solely or in combination. ${ }^{9}$ Obvious advantages of MTs could be the rapid removal of the thrombus without the need for prolonged lytic infusions, which is known for potential life threatening bleeding complications. Hilliard et al..$^{10}$ recently described 

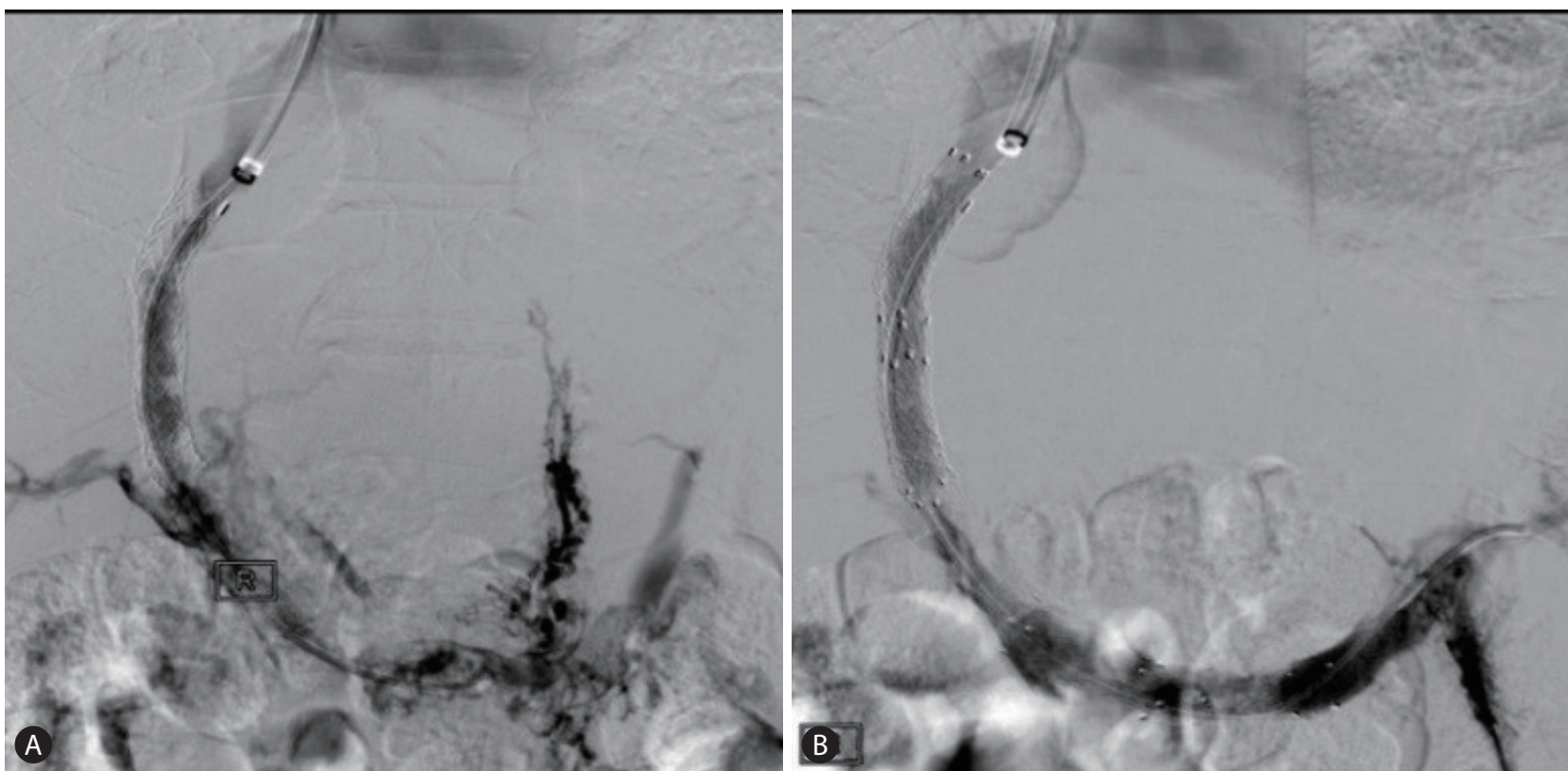

Figure 3. (A) Venography after mechanical and suction thrombectomy shows partial recanalization of TIPS tract and main portal vein with contrast media filling in the collateral veins. (B) Venography after angioplasty plus stenting demonstrates a rapid contrast medium outflow toward the shunt and the hepatic veins without visualization of the collateral veins. TIPS, tansjugular intrahepatic portosystemic shunt.

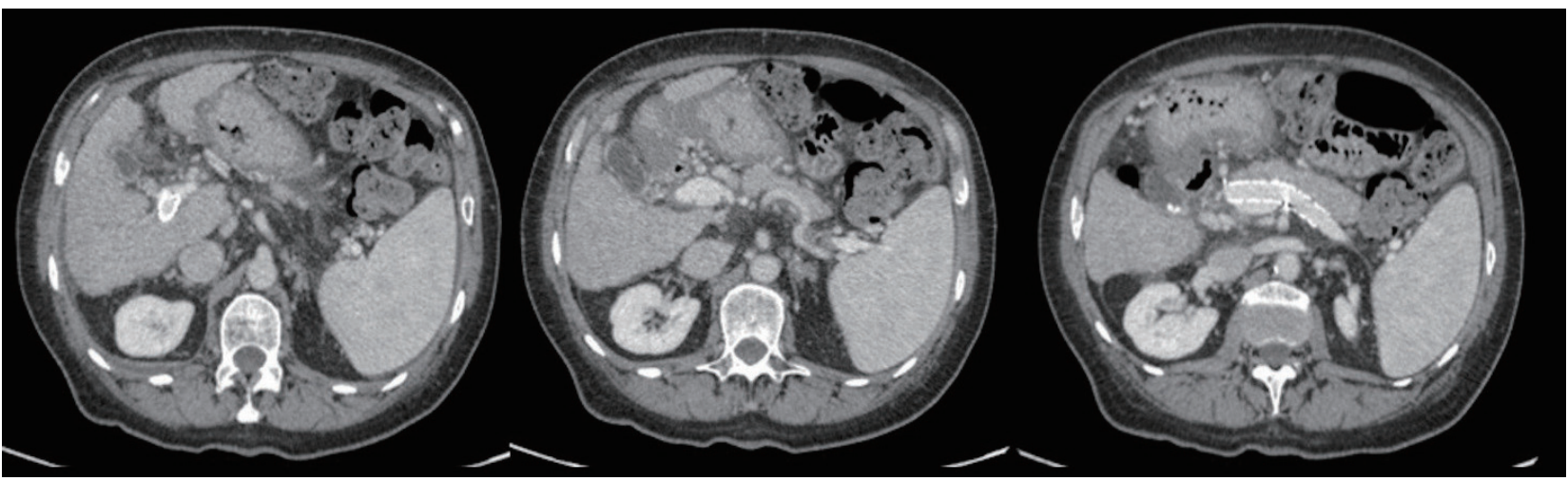

Figure 4. A computed tomography scan performed 6 months after the procedure showing the complete resolution of the thrombosis of the shunt, the intrahepatic portal vein branches and the main portal trunk up to the spleno-mesenteric confluence.

the use of US-assisted thrombolysis for TIPS occlusion, demonstrating decreased risks associated with thrombolytic agents shortening treatment times. However, this technique requires additional equipment required compared to the standard catheterdirect thrombolysis and is therefore more expensive. In our case an subacute TIPS thrombosis extended into the portal, splenic and SMV, occurred in a cirrhotic decompensated patient (Child Pugh class B and with grade III ascites), which has been treated with the jugular vein approach and thrombectomy in combination with three mechanical techniques: mechanical and suction thrombec- tomy, following angioplasty plus stenting. This approach may be useful approach to treat TIPS occlusion where single conventional techniques might be ineffective or where thrombolytic therapy is contraindicated.

\section{Authors' contributions}

Study concept and design: NDM, CDS, LM. Data acquisition: NDM, FP. Data analysis and interpretation: NDM, CDS, LM. Drafting of the manuscript; critical revision of the manuscript for important intellectual content: NDM, CDS, LM. Study supervision: 
RM, GR, LM. All authors approved the final version of the manuscript.

\section{Acknowledgements}

The authors are indebted to Mrs. Franziska Michaela Lohmeyer for language editing, and proofreading.

\section{Conflicts of Interest}

The authors have no conflicts to disclose.

\section{REFERENCES}

1. Neong SF, Adebayo D, Wong F. An update on the pathogenesis and clinical management of cirrhosis with refractory ascites. Expert Rev Gastroenterol Hepatol 2019;13:293-305.

2. Boyer TD, Haskal ZJ; American Association for the Study of Liver Diseases. The role of transjugular intrahepatic portosystemic shunt (TIPS) in the management of portal hypertension: update 2009. Hepatology 2010;51:306.

3. Yue-Meng W, Li YH, Wu HM, Yang J, Yang LH, Xu Y. Portal vein thrombosis in patients with cirrhosis undergoing elective transjugular intrahepatic portosystemic shunt: risk factors, warfarin efficacy, and clinical outcomes. Clin Appl Thromb Hemost 2018;24:462-470.

4. Miraglia R, Maruzzelli L, Luca A. Recanalization of occlusive transjugular intrahepatic portosystemic shunts inaccessible to the standard transvenous approach. Diagn Interv Radiol 2013;19:61-65.

5. Pereira K, Baker R, Salsamendi J, Doshi M, Kably I, Bhatia S. An approach to endovascular and percutaneous management of transjugular intrahepatic portosystemic shunt (TIPS) dysfunction: a pictorial essay and clinical practice algorithm. Cardiovasc Intervent Radiol 2016;39:639-651.

6. Haskal ZJ, Duszak R Jr, Furth EE. Transjugular intrahepatic transcaval portosystemic shunt: the gun-sight approach. J Vasc Interv Radiol 1996;7:139-142.

7. Cura M, Cura A, Suri R, El-Merhi F, Lopera J, Kroma G. Causes of TIPS dysfunction. AJR Am J Roentgenol 2008;191:1751-1757.

8. Uflacker R. Mechanical thrombectomy in acute and subacute thrombosis with use of the Amplatz device: arterial and venous applications. J Vasc Interv Radiol 1997;8:923-932.

9. Casanegra $A l, M c B a n e ~ R D$, Bjarnason $H$. Intervention radiology for venous thrombosis: early thrombus removal using invasive methods. Br J Haematol 2017;177:173-184.

10. Hilliard N, See TC, Shaida N. Ultrasound-assisted thrombolysis of an occluded transjugular portosystemic shunt. Diagn Interv Radiol 2017;23:318-320. 